\title{
Supporting Software Process Tracking Through the Internet
}

\author{
Joseph E. Urban and Shriram Sankaran \\ Arizona State University \\ Department of Computer Science and Engineering \\ Tempe, AZ 85287-5406 USA
}

\begin{abstract}
The Internet is becoming a medium for distributed software development groups. An undergraduate two-course capstone sequence, Software Engineering Project I and II uses the Internet in order to accomplish many tasks that support software development. This paper includes a description of the course demographics and the use of the Internet in carrying out project development activities. The SPAWS software tool is an Internet based software tool for project management that was developed in order to support these courses. The paper includes an overview of the tool and an evaluation of the tool capabilities.
\end{abstract}

Key words: course project, distributed software development, Internet based tool, project management, software process tracking, software tool

\section{INTRODUCTION}

The courses, Software Engineering Project I and II, offer an opporturity for students to obtain experience in the way that the software industry functions while the students are still at school. Software Engineering Project I students perform in small teams (of less than 6 members each) to go through the early stages of the software life cycle of medium size software projects namely, project proposal, requirements specification, design, coding of a selected module for the project, validation of all the software development products. Software Engineering Project II requires the students of the class to perform as a single group to select a project from the list 
designed in the previous course, perform any design changes if necessary and go through the later stages of the software lifecycle namely, complete development, testing, and maintenance. This course also provides the students an opportunity to experience first hand the object-oriented development principles, Unified Modeling Language (UML), CASE tools, Capability Maturity Model (CMM), and configuration management.

The Internet has made possible for geographically separated software programmers working on the same project to be in synchronization with each other [Ahamed2000, Cusumano1999a, Cusumano1999b, Urban1999]. Software engineers can follow process activities no matter where they work. The SPAWS (Software Process Automation and Workflow System) [Sankaran2002], was designed to allow software project members working on the same project to follow the process activities.

The Software Engineering Project I offered during the Spring 2001 semester produced eight different project designs for design tools based on different techniques. The Software Engineering Project II offered during the Fall 2001 semester, implemented the "E-modeler" project, based on the object modeling technique developed by Rumbaugh [Rumbaugh1991]. This project had 19 students working on the same project in 4 groups - server, database, user interface, and graphics. In order to accomplish the Software Engineering Institute's Capability Maturity Model (CMM) level 4, the group had to design process activities and produce process documents [Paulk1993]. A need was generated to implement a software system that would accomplish automating the processes for ease of use by the members involved. This need was the motivation for the SPAWS project.

The remainder of this paper consists of background on the software development setting that lead to the software requirements for SPAWS. The next major section describes SPAWS to include features, tasks, and the software architecture. There is an evaluation of SPAWS in the context of an Internet based tool for software process tracking. The paper concludes with a summary and future research section.

\section{A TWO COURSE SOFTWARE ENGINEERING PROJECT CAPSTONE SEQUENCE}

The purpose of Software Engineering Project I course is to provide students with hands-on experience in planning and managing a medium size software project in a team environment using object-oriented software development principles, the Unified Modeling Language (UML), a variety of CASE tools, Capability Maturity Model (CMM) guidelines, and applying configuration management. There are topics covered in the course which are 
related to large-scale software development, such as process definition guidelines, and how risk management is practiced throughout project development [Pfleeger1998]. The ACM / IEEE Computer Society software engineering code of ethics is covered through lecture and group activities [Gotterbarn1999].

The software engineering student project teams who work on objectoriented analysis and design of a web based IEEE standard software engineering tool with an extensive use of CASE tools and the use of software packages. The capability maturity model guidelines, risk management, and software quality assurance are used to continuously improve the quality of the group effort. One module of the software design is carried through code implementation and testing. The project is later finished in the Software Engineering Project II course with implementation, testing, and evaluation.

The Software Engineering Project II course provides the students with a hands-on team-work experience in planning, managing, and developing a medium size web based software product in a self directed team environment using various software engineering principles and CASE tools. The students use the object-oriented analysis and design documents, which were prepared by the Software Engineering Project I students during the previous Spring semester, as the basis for implementing the software product. The students learn to use incremental software development techniques, object-oriented programming styles, distributed software development and testing methodologies throughout the project development. The topics that were covered in the Software Engineering Project I course (e.g., process definition, risk management, object-oriented analysis and design, and CASE tools) are used in the group project. Additional topics, such as software quality, software maturity model and outsourcing are also addressed.

During Software Engineering Project II, teams of students work on the implementation, testing, and modification of a medium size distributed object-based software product using web-based implementation tools. The students use the object-oriented analysis and design documents prepared by the Software Engineering Project I teams during the previous Spring semester. Each team is responsible for preparing a tentative milestone calendar with risk identification documents, collaboration of distributed development groups and necessary testing plans, which are followed throughout the development phase.

The students in the courses are physically distributed throughout the greater Phoenix metropolitan area. The geographical dispersion combined with varying class and work schedules makes face-to-face meeting sometimes difficult. However, there is some use of in-class time for the conducting of group meetings, as well as mutually agreed upon times while 
on campus. For the most part, the students depend on the Internet as a means to meet and communicate with each other.

The University provides support for group activities at a moderate level with physical meeting space, e.g., in unused classrooms, the library, and specially designated rooms in the main computing facility. Although not the equivalent of having cubicle space in a setting of a high technology firm, there are plans to pursue more extensive meeting facilities. However, the emerging manner of distributed members of a software development effort may preclude the need for extensive physical facilities in favor of a comprehensive virtual meeting space.

Now the University provides a virtual classroom capability through an implementation of BlackBoard under a myASU portal. This approach allows group forums to be established that provide for e-mail, file transfer, and chat rooms. In addition, some groups have taken to other commercial Internet group sites, such as Yahoo! groups. Software Engineering Project II course used this approach during the Fall 2001 semester. There was degradation of service with myASU due to increased volume. Rather than wait for the improvement in performance, the Yahoo! groups approach was adopted and maintained even when the University service reached an acceptable level.

The course project was based on the ANSI/IEEE Std. 1016-1987 (Reaffirmed 1993), IEEE Recommended Practice for Software Design Descriptions [IEEE1997c], and ANSI/IEEE Std. 1016.1-1993, IEEE Guide to Software Design Descriptions [IEEE1997d]. Other versions of the course project have been based on the ANSI/IEEE Std. 830-1993, IEEE Recommended Practice for Software Requirements Specifications [IEEE1997a], ANSI/IEEE Std. 1058.1-1987 (Reaffirmed 1993), IEEE Standard for Software Project Management Plans [IEEE1997b], and IEEE Std. 829-1998, IEEE Standard for Software Test Documentation [IEEE1998] that was developed by the Life Cycle Data Harmonization Working Group of the Software Engineering Standards Committee of the IEEE Computer Society.

The project was a group effort that involved the development of the software requirements, user guide, risk management, design, code, and testing for a software tool to support web-based software design documents formulation, analysis, storage / retrieval, and reporting that is based on the standard. A different type of design document was assigned to each group for representation in the software tool. A context sensitive editor that assists software engineers in constructing and updating software design documents may or may not have been integrated with the analysis part of the tool that checks for consistency and completeness. The storage and retrieval part of the software tool provided for save and search of software design documents. The reporting part of the software tool provided a printed 
version for any part of a design document. In the Software Engineering Project I course each group developed tool support from requirements through design for the complete software design documents tool and implemented an aspect of executable code for the tool. In the Software Engineering Project II course the class completed the design, implementation, and testing of the software tool.

The Software Engineering Project II course members conducted a selfassessment of the Capability Maturity Model in order to determine a level of performance and to use as a basis for managing the project. Each group responded with yes, no, or maybe, as well as provided a justification for the answer. For example in the key process areas for Level 2, repeatable, there is a key process area on Software Project Tracking \& Oversight, which has the goal: "Goal 3 Changes to software commitments are agreed to by the affected groups and individuals." The groups responded to these goals as follows: Graphics - "Yes, the UI and Graphics team enjoy good communication", UI - "Yes, all groups and the customer agreed to changes in the commitments", Database - "Yes - although this happens at the last minute sometimes", and Server - "yes: we meet with DB group to confirm this."

By the end of the semester, the class had done well to achieve level 3, defined, which was based on the self-assessment. There were several level 4, managed, key process area goals that were met through the self assessment, but even less level 5, optimizing, goals. In general, the exercise was most beneficial as a management tool for putting processes and procedures in place, but more importantly, this effort was the primary driver behind developing the SPAWS software requirements.

This section has provided an overview of the two-course capstone sequence that lead to the development of SPAWS. This overview described both of the courses, the course project, and capability maturity model analysis. The next section is a description of the SPAWS web based software tool that was developed to support the Software Engineering Project courses.

\section{SPAWS}

SPAWS is a software system that allows software engineers working on the same project to follow process activities. The SPAWS system currently has three process activities implemented - document inspection, code inspection, and document review. However, the system has been designed in such a way that any other process activity may be automated. 


\subsection{Features of SPAWS}

The features displayed for a user in the SPAWS system are:

- Login: the user may log into the system using a login name and password. Authentication is done by the server (Controller);

- New task: the user may initiate new process activities from the list of activities of document review, code review, and code inspection. The user may type in English using the keyboard in the text area provided for the description of the task;

- Pending task: the user may select a particular task from the list of pending tasks, may perform the task, and include comments for the task;

- Old tasks: the user may select this option to view the ten most recent tasks performed; and

- Logout: the user may logout of the system.

The user defined as the moderator for a particular task has the following options:

- moderator may select the participants involved in an activity, namely the inspectors/reviewers, reader and recorder.

\subsection{Task details}

The user initiating a task is called the author.

Code inspection: the user may select the code inspection type of task when initiating a new task/activity:

- user may select the moderator;

- moderator may select the reader, recorder and inspectors;

- reader and the inspectors may inspect the code and write comments ;

- recorder may record final comments about the code inspected in the format provided;

Document inspection: the user may select the document inspection type of task when initiating a new task/activity:

- user may select the moderator;

- moderator may select the reader, recorder and inspector;

- reader and the inspectors may inspect the document and write comments;

- recorder may record final comments about the document inspected in the format provided;

Document review: the user may select the document review type of task when initiating a new task/activity:

- user may select the reviewers;

- inspectors may review the document;

- inspectors may record comments about the document reviewed in the format provided; 


\subsection{Program Structure}

A detailed description of the program structure used in the software is presented in this section. A pictorial representation of the architecture is presented below:

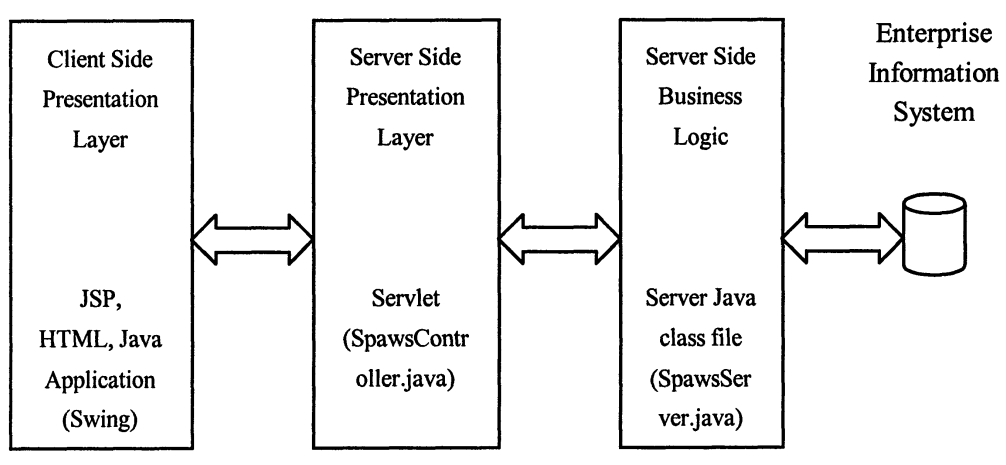

Figure 1. J2EE Application model [Sun2002]

\subsection{Description of components}

This sub-section provides a description of each of the components identified in the architecture diagram above.

\section{Client side presentation layer:}

This layer may be implemented as follows:

1. Application: a Swing application that connects to the Server side presentation layer through http protocol. (not in the scope of project)

2. Java Server Pages: a set of JSP pages, which displays a mix of static and dynamic web content on the same web page.

This layer records user responses and transmits them to the server side presentation layer for further processing. Client side presentation layer also displays the results of a transaction in a meaningful form easily understood by the user. 


\section{Server side presentation layer:}

This layer essentially has a Servlet - "SpawsController", that receives the user responses from the Client side presentation layer and then based on the internal logic, processes the request. The server side presentation layer connects to the database through the Server side business logic layer. If the request is to direct to a different screen, then the servlet does so.

\section{Server side business logic:}

This layer contains the Server - "SpawsServer", which processes the database request from the presentation layer and then processes the request by connecting to the database through the Enterprise information system. In case the number of connections to the database is less than the requests for connections, then a queue is maintained for the requests not being processed and then connections are allotted to the server, based on the time of receiving the request.

\section{Enterprise information system:}

This layer contains the "ConnectionPool", which manages a static number of database connections. The enterprise information system layer is responsible for allocating a connection when requested by the server side business logic and then releasing the connection when the transaction has been processed.

\subsection{GUI Components}

The GUI components used for implementation are:

- Login.jsp: to display the login screen for the user;

- Status.jsp: to display the status information for the user including the number of pending tasks and the login status;

- CreateTask.jsp: to create a new task;

- CreateDocument.jsp: to create a document when a task is created;

- SelectParticipants.jsp: to select the participating users for the task created;

- PendingTasks.jsp: to display brief information about the pending tasks for the user;

- SelectDocument.jsp: to select the document to be reviewed when a pending task is selected;

- OpenDocument.jsp: to display the document to be reviewed for a pending task; 
- ReviewDocument.jsp: to enter the comments or report of review;

- OldTasks.jsp: to display the ten most recent tasks completed by a user; and

- InformationScreen.jsp: to change any information about a user.

\subsection{Tier description}

The jsp files, which act as the "View", would be invoked by the "SpawsController.java" which is a servlet and which performs the "Controller" function as defined in the J2EE specifications. This servlet is resident on the servlet container and handles all user interaction and directs control to the respective jsp pages for processing. This servlet also communicates to the database through the "Model" tier, which is the "DBComm.java" file.

The "Controller" tier consists of "SpawsController.java" file. The main function of this file is to receive user input from the GUI and interpret it to the database as actions. This tier also focuses on redirecting the user through the different jsp pages.

The "DBComm.java" connects to the database through the "ConnectionPool.java", which ensures that only a limited number of connections are available to the database. ConnectionPool also manages the allocation of free connections and releasing the connections, which are no longer in use. The ConnectionPool ensures that no matter how many simultaneous connections the database can support, the number of connections are never above the maximum limit supported.

\section{EVALUATION OF SPAWS}

This section describes an evaluation the SPAWS system and the special features of the system.

\section{Operating system independence}

Since this software was developed in Java, it is operating system independent. It can run on any computer irrespective of the environment provided the basic environment setup is satisfied.

\section{Modularity}

The SPAWS software was developed in a modular fashion using the MVC architecture of J2EE specifications [Sun2002]. This approach makes software maintenance easy, which means that in most cases, only necessary 
layers have to be altered. Any new modules may be added without many alterations to the other layers.

\section{Easy user interface}

The user interface has been designed using Java Server Pages (JSP). The UI programmer may change the look and feel of the user interface without having to know much of the Java programming language.

\section{Pure Java compatible}

This software has been developed completely in the Java language. Even the database being used, Cloudscape, is a $100 \%$ Pure Java application. Hence, this application conforms to the specifications of a Pure Java application.

\section{J2EE Compliant}

This software has been modeled based on the MVC architecture as specified in the J2EE model. [Sun2002]

\section{Web-enabled}

This software is web-enabled. This software can be accessed from any part of the globe by using an Internet browser.

\section{SPAWS: LESSONS LEARNED}

The experiences encountered during the course of SPAWS software development have been summarized below:

\section{Schedule}

Deadlines and weekly progress meetings ensured that the project was on track. Since only one person was involved in the development, the deadlines provided a motivation for project completion.

\section{Communication}

E-mail and weekly progress meetings ensured that the project requirements matched those of the customer.

\section{Design}

In order to convey the design of the software, documents and architecture diagrams were used. Proper documentation made the documents available for future use for software maintenance. 


\section{Prototype}

A prototype of the project developed early on in the project helped in communicating the user interface to the customer and the development phase was started only after approval of the prototype. This made sure that the customer requirements were communicated to the developer completely without any ambiguity.

\section{Documentation}

The use of extensive documentation throughout the project ensured that the customer and developer agreed on the same requirements and also to help in post-mortem analysis of the project.

\section{Version control}

Version control was an important aspect of the SPAWS project, since three versions of the software were released. Maintaining the three versions with version control made it more organized.

\section{SUMMARY AND FUTURE WORK}

This paper provided an overview of a two-course capstone sequence, Software Engineering Project I and II. This discussion included the course demographics and the use of the Internet in carrying out project development activities. The SPAWS software tool is an Internet based software tool for project management that is an outgrowth of these courses.

The SPAWS software tool has been made available to the Department of Computer Science and Engineering in Arizona State University and hence is available for the current students to be used for academic purposes. Features can be added or deleted to the software and more user interfaces can be added based on the latest Internet access gadgets available. Also more process activities could be automated in a step towards total workflow automation.

Additionally, this software may also have the user interface layer coded as an application instead of jsp pages for ease of use. The other layers still may remain the same. Other options available for future enhancements are the features available for editing the document attached for review / inspections and the use of XML document for data storage and representation.

The future offerings of the Software Engineering Project I and II courses will be using the SPAWS system to follow process activities. Based on tool use and additional self-assessments of the capability maturity model, there 
will be enhancements to the SPAWS software requirements. These changes will be reflected in the tool.

\section{REFERENCES}

[Ahamed2000] S. I. Ahamed, S. Ali, D. G. Bingham, A. Dawra, L. T. Ha, T. M. Luong, D. M. Martinez, J. Morris, S. A. Palangala, and J. E. Urban, "Software Requirements on the Web," Proceedings of the $4^{\text {th }}$ International Conference on Business Information Systems (BIS'2000), Poznan, Poland, April 12-13, 2000, pp.134-144.

[Cusumano1999a] M. A. Cusumano and D. B. Yoffie, "What Netscape Learned from CrossPlatform Software Development," Communications of the ACM, Vol. 42, No. 10, October 1999 , pp. $72-78$.

[Cusumano1999b] M. A. Cusumano and D. B. Yoffie, "Software Development on Internet Time," Computer, Vol. 32, No. 10, October 1999, pp. 60-69.

[Gotterbarn1999] D. Gotterbarn, K. Miller, and S. Rogerson, "Software Engineering Code of Ethics is Approved," Communications of the ACM, Vol. 42, No. 10, October 1999, pp. 102-107.

[IEEE1997a] IEEE, “ANSI/IEEE Std. 830-1993, IEEE Recommended Practice for Software Requirements Specifications," in IEEE Standards Collection: Software Engineering, IEEE, New York, New York, 1997.

[IEEE1997b] IEEE, “ANSI/IEEE Std. 1058.1-1987 (Reaffirmed 1993), IEEE Standard for Software Project Management Plans," in IEEE Standards Collection: Software Engineering, IEEE, New York, New York, 1997.

[IEEE1997c] IEEE, “ANSI/IEEE Std. 1016-1987 (Reaffirmed 1993), IEEE Recommended Practice for Software Design Descriptions," in IEEE Standards Collection: Software Engineering, IEEE, New York, New York, 1997.

[IEEE1997d] IEEE, “ANSI/IEEE Std. 1016.1-1993, IEEE Guide to Software Design Descriptions," in IEEE Standards Collection: Software Engineering, IEEE, New York, New York, 1997.

[IEEE1998] IEEE, "IEEE Standard for Software Test Documentation," IEEE, New York, New York, 1998

[Paulk1993] M.C. Paulk, B. Curtis, M. B. Chrissis, and C. V. Weber, Capability Maturity Model for Software, Version 1.1, Software Engineering Institute, Technical Report, CMU/SEI-93-TR-024, ESC-TR-93-177, February 1993, 82 pp.

[Pfleeger1998] S. L. Pfleeger, Software Engineering: Theory and Practice, Prentice-Hall, Inc., Upper Saddle River, NJ, 1998.

[Rumbaugh1991] J. Rumbaugh, "Object-Oriented Modeling and Design”, Prentice Hall, 1991.

[Sankaran2002] S. Sankaran, SPAWS - Software Process Automation and Workflow System, MCS Project Report, May 2002, 44 pp.

[Sun2002] Sun Microsystems, "Designing Enterprise Applications with the J2EE Platform," $\mathrm{http}: / / j$ java.sun.com/blueprints/guidelines/designing_enterprise_applications/index.html

[Urban1999] J. E. Urban, "Software Engineering on the Web," Proceedings of the $3^{\text {rd }}$ International Conference on Business Information Systems, Poznan, Poland, April 14-16, 1999, pp. 113-122. 